\title{
The Determinant of Pentadiagonal Centrosymmetric Matrix Based on Sparse Hessenberg's Algorithm
}

\author{
N Khasanah 1,* \\ ${ }^{1}$ Mathematics Department, Faculty of Science and Technology, UIN Walisongo Semarang, Indonesia
${ }^{*}$ Corresponding author. Email: khasanah.nur@walisongo.ac.id
}

\begin{abstract}
The algorithm of general pentadiagonal matrix has been evaluated before for computational purpose. The properties of this matrix on sparse structure are exploited to compute an efficient algorithm. This article propose a new construction of pentadiagonal matrix having centrosymmetric structure called pentadiagonal centrosymmetric matrix. Moreover, by applying the algorithm of determinant sparse Hessenberg matrix, an explicit formula of pentadiagonal centrosymmetric matrix's determinant is developed.
\end{abstract}

Keywords: general pentadiagonal, centrosymmetric structure, sparse Hessenberg, algorithm, determinant.

\section{INTRODUCTION}

Pentadiagonal matrix is the one construction of sparse matrix widely applied in areas of science and engineering, such in numerical solution of ordinary and partial differential equations (ODE and PDE), interpolation problems and boundary value problems (BVP) [1]. The rule of this matrix is necessary at many areas, then the evaluation of this matrix are needed, particularly at determinant process. Based on the special structure of this matrix, some researchers focus on determinant problem based on computation stand point.

The main basic research about pentadiagonal matrix is started by [2] using two-term recurrence for evaluating determinant general matrix. Based on previous research [3] at computing the determinant of a tridiagonal matrix, generalization of the DETGTRI algorithm are obtained. This algorithm as the major concept for the next discussion on constructing determinant of pentadiagonal matrix [4-11].

On the other side, centrosymmetric matrix also has a special structure arise at some applications, for instance pattern recognition process [12]. By using analytical process this special entries of centrosymmetric matrix, the computation of determinant matrix is important to be evaluated. Studies about this topics are given at some papers [13-16] for a number of fast algorithm for computing determinant process by applying Hessenberg algorithm of determinant. This kind of matrix having rules on numerical analysis and arise at determinant centrosymmetric matrix [17-18].
The aim of this paper is to construct a new form of pentadiagonal matrix with centrosymmetric structure. Due to application both matrices and evaluation the structure, the algorithm of determinant of this matrix is proposed by using Hessenberg rule.

\section{PRELIMINARIES}

First of all, some definitions and properties are given for clear discussion at determinant process of pentadiagonal centrosymmetrix matrix, as follows.

Definition 1 [11]. The matrix is called as $n \times n$ general pentadiagonal matrix which has definition such $\mathbf{D}=\left(d_{i j}\right)_{1 \leq i, j \leq n}$, where the entry $d_{i j}=0$ for $|i-j|>2$ or can be written as

$$
\mathbf{D}=\left(\begin{array}{ccccccc}
d_{11} & d_{12} & d_{13} & & & & \\
d_{21} & d_{22} & d_{23} & d_{24} & & & \\
d_{31} & d_{32} & d_{33} & d_{34} & d_{35} & & \\
& \ddots & \ddots & \ddots & \ddots & \ddots & \\
& & \ddots & \ddots & \ddots & \ddots & d_{n-2, n} \\
& & & d_{n-1, n-3} & d_{n-1, n-2} & d_{n-1, n-1} & d_{n-1, n} \\
& & & & d_{n, n-2} & d_{n, n-1} & d_{n, n}
\end{array}\right)
$$


Definition 2 [13]. The entries of $n$-by- $n$ lower Hessenberg matrix is the form as follows

$$
\mathbf{H}=\left(\begin{array}{ccccc}
h_{11} & h_{1,2} & 0 & 0 & 0 \\
h_{21} & h_{2,2} & h_{2,3} & 0 & 0 \\
\vdots & \vdots & \ddots & \ddots & 0 \\
h_{n-1,1} & h_{n-1,2} & \cdots & h_{n-1, n-1} & h_{n-1, n} \\
h_{n, 1} & h_{n, 2} & \cdots & h_{n, n-1} & h_{n, n}
\end{array}\right)
$$

Definition 3 [15]. The $n$ order of sparse Hessenberg matrix is the matrix with the contruction as

$$
\mathbf{S}=\left(\begin{array}{ccccccc}
h_{11} & h_{1,2} & h_{1,3} & & & & \\
h_{21} & h_{2,2} & h_{2,3} & h_{2,4} & & & \\
& h_{3,2} & h_{3,3} & h_{3,4} & h_{3,5} & & \\
& & \ddots & \ddots & \ddots & \ddots & \\
& & & \ddots & \ddots & \ddots & h_{n-2, n} \\
& & & & h_{n-1, n-2} & h_{n-1, n-1} & h_{n-1, n} \\
& & & & & h_{n, n-1} & h_{n, n-}
\end{array}\right)
$$

Definition 4 [14]. The centrosymmetric matrix is the matrix where $\mathbf{A}=\left(a_{i j}\right)_{n \times n} \in R^{n \times n}$ and has the entries as $a_{i j}=a_{n-i+1, n-j+1}$ for $1 \leq i \leq n, 1 \leq j \leq n$ or

$$
\mathbf{A}=\left(\begin{array}{cccc}
a_{11} & a_{12} & \cdots & a_{1, n} \\
a_{21} & a_{22} & \cdots & a_{2, n} \\
\vdots & \vdots & \vdots & \vdots \\
a_{2, n} & \cdots & a_{22} & a_{21} \\
a_{1, n} & \cdots & a_{12} & a_{11}
\end{array}\right)
$$

\section{RESULTS AND DISCUSSION}

This section discuss about the main result of this paper on constructing a new form of pentadiagonal centrosymmetric matrix. Then, the algorithm to compute determinant of this matrix is proposed also. Based on the rule of sparse Hesssenberg matrix, the steps of this algorithm is explained for deeper understanding

The construction of general pentadiagonal matrix and its algorithm for computing determinant have been found by [4]. As the different point of view from general structure of pentadigonal matrix, this article shows a new construction pentadiagonal matrix having centrosymmetric structure. The formation of this matrix we called as pentadiagonal centrosymmetric matrix. After constructing new form of the matrix, next we derive numerical algorithm for computing this new matrix. Based on the algorithm of sparse Hessenberg, the explanations of this algorithm are written as follows.

First, let consider transforming the general pentadagonal matrix (1) into the formation of pentadiagonal centrosymmetric matrix. In this discussion, we focus on general pentadiagonal matrix, where is even number as its matrix ordo only. Based on the definition before about general pentadiagonal (1) and centrosymmetric matrix (4), the formationpentadiagonal centrosymmetric matrix is formed by the step:

\subsection{Construct Sparse Hessenberg Matrix}

Based on definition of pentadiagonal and centrosymmetric matrix, then the pentadiagonal centrosymmetric matrix is written as (1) where the entries have centrosymmetric structure $d_{i j}=d_{n-i+1, n-j+1}$ for $1 \leq i \leq n, 1 \leq j \leq n$. For a deeper understanding, let we show an example the form of $8 \times 8$ pentadiagonal matrix to be the specific matrix called pentadiagonal centrosymmetric matrix such :

$$
\mathbf{D}_{8}=\left(\begin{array}{lllllllll}
d_{11} & d_{12} & d_{13} & & & & & \\
d_{21} & d_{22} & d_{23} & d_{24} & & & & \\
d_{31} & d_{32} & d_{33} & d_{34} & d_{35} & & & \\
& d_{42} & d_{43} & d_{44} & d_{45} & d_{56} & & \\
& & d_{53} & d_{54} & d_{55} & d_{56} & d_{57} & \\
& & & d_{64} & d_{65} & d_{66} & d_{67} & d_{68} \\
& & & & d_{75} & d_{76} & d_{77} & d_{78} \\
& & & & & d_{86} & d_{87} & d_{88}
\end{array}\right) \text { is }
$$

pentadiagonal form matrix. Then it constructed to be pentadiagonal having centrosymmetric structure such

$$
\tilde{\mathbf{D}}_{8}=\cdot\left(\begin{array}{lllllllll}
d_{11} & d_{12} & d_{13} & & & & & \\
d_{21} & d_{22} & d_{23} & d_{24} & & & & \\
d_{31} & d_{32} & d_{33} & d_{34} & d_{35} & & & \\
& d_{42} & d_{43} & d_{44} & d_{45} & d_{46} & & \\
& & d_{46} & d_{45} & d_{44} & d_{43} & d_{42} & \\
& & & d_{35} & d_{34} & d_{33} & d_{32} & d_{31} \\
& & & & d_{24} & d_{23} & d_{22} & d_{21} \\
& & & & & d_{13} & d_{12} & d_{11}
\end{array}\right)
$$

After getting this form matrix, the next work is transforming pentadiagonal into sperse Hessenberg matrix. This step is usefull for applying Hesssenberg algorithm at determinant computation. Based on the definition of pentadiagonal form (1), then constructing with centrosymetric entries, it continues by constructing sparse Hessenberg matrix $\mathbf{H}=\left(d_{i j}\right)_{1 \leq i, j \leq n}$ written as [4]

$$
\mathbf{H}=\left(\begin{array}{ccccccc}
d_{11} & d_{12} & d_{13} & & & & \\
d_{21} & d_{22} & d_{23} & d_{24} & & & \\
& d_{32} & d_{33} & d_{34} & d_{35} & & \\
& & \ddots & \ddots & \ddots & \ddots & \\
& & & \ddots & \ddots & \ddots & d_{n-2, n} \\
& & & & d_{n-1, n-2} & d_{n-1, n-1} & d_{n-1, n} \\
& & & & & d_{n, n-1} & d_{n, n}
\end{array}\right)
$$


From the above matrix, we choose $\lambda_{\mathbf{i}+1, \mathbf{i}}$ from the matrix of

$$
\lambda_{i+1}=\left(\begin{array}{cccc}
I_{i-1} & & & \\
& 1 & & \\
& \lambda_{i+1, i} & 1 & \\
& & & I_{n-i-1}
\end{array}\right)
$$

As the ilustration, for $8 \times 8$ pentadiagonal centrosymmetrix matrix (5) then by choosing $\lambda_{3,2}=-\frac{d_{3,1}}{d_{2,1}}$ to transform matrix where :

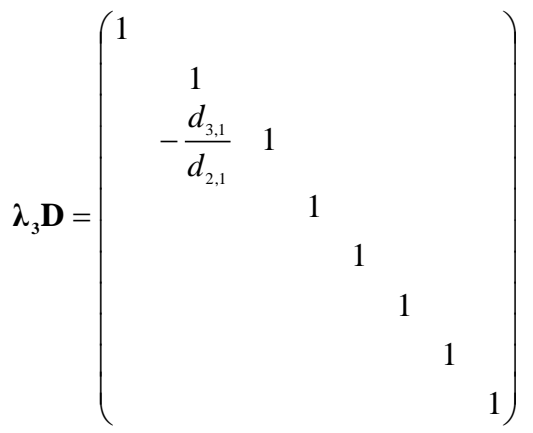$$
\cdot\left(\begin{array}{llllllll}
d_{11} & d_{12} & d_{13} & & & & & \\
d_{21} & d_{22} & d_{23} & d_{24} & & & & \\
d_{31} & d_{32} & d_{33} & d_{34} & d_{35} & & & \\
& d_{42} & d_{43} & d_{44} & d_{45} & d_{46} & & \\
& & d_{46} & d_{45} & d_{44} & d_{43} & d_{42} & \\
& & & d_{35} & d_{34} & d_{33} & d_{32} & d_{31} \\
& & & & d_{24} & d_{23} & d_{22} & d_{21} \\
& & & & & d_{13} & d_{12} & d_{11}
\end{array}\right)
$$$$
=\left(\begin{array}{llllllll}
d_{11} & d_{12} & d_{13} & & & & & \\
d_{21} & d_{22} & d_{23} & d_{24} & & & & \\
& \tilde{d}_{32} & d_{33} & d_{34} & d_{35} & & & \\
& d_{42} & d_{43} & d_{44} & d_{45} & d_{46} & & \\
& & d_{46} & d_{45} & d_{44} & d_{43} & d_{42} & \\
& & d_{35} & d_{34} & d_{33} & d_{32} & d_{31} \\
& & & d_{24} & d_{23} & d_{22} & d_{21} \\
& & & & & d_{13} & d_{12} & d_{11}
\end{array}\right) .
$$

Repeating the same process, by taking $\lambda_{4,3}=-\frac{d_{42}}{d_{32}}, \cdots, \lambda_{12}=-\frac{d_{13}}{d_{23}}$ and the end step resulting the following form of matrix.

$$
\lambda_{8} \lambda_{7} \lambda_{6} \cdots \lambda_{3} \mathbf{D}=\left(\begin{array}{llllllll}
d_{11} & d_{12} & d_{13} & & & & \\
d_{21} & d_{22} & d_{23} & d_{24} & & & \\
& \tilde{d}_{32} & \tilde{d}_{33} & \tilde{d}_{34} & d_{35} & & & \\
& & \tilde{d}_{43} & \tilde{d}_{44} & \tilde{d}_{45} & d_{56} & & \\
& & & \tilde{d}_{45} & \tilde{d}_{44} & \tilde{d}_{43} & d_{42} & \\
& & & & \tilde{d}_{34} & \tilde{d}_{33} & \tilde{d}_{32} & d_{31} \\
& & & & & \tilde{d}_{23} & \tilde{d}_{22} & \tilde{d}_{21} \\
& & & & & & \tilde{d}_{12} & \tilde{d}_{11}
\end{array}\right)
$$

For a general formula, the number of $\lambda_{i+1, i}$ is defined as $\lambda_{i+1, i}=-\frac{d_{i+1, i-1}}{\tilde{d}_{i, i-1}}$ where $\tilde{d}_{21}=d_{21}$. By the concept of $\mathbf{V}=\lambda_{n} \lambda_{n-1} \cdots \lambda_{3}$, it has the form of $\mathbf{V D}=\mathbf{H}$ [14]. Therefore,

$\operatorname{det}(\mathbf{D})=\operatorname{det}(\mathbf{V}) \cdot \operatorname{det}(\mathbf{D})=\operatorname{det}(\mathbf{V D})=\operatorname{det}(\mathbf{H})$, with the $\operatorname{det}(\mathbf{V})=1$

Based on the element of $\mathbf{D}$ which integer numbers or $\mathbf{d}_{\mathbf{i j}} \in \mathbf{Z}$, then it can use the following matrix

$\lambda_{i+1}=\left(\begin{array}{cccc}I_{i-1} & & & \\ & 1 & & \\ & -d_{i+1, i-1} & \tilde{d}_{i, i-1} & \\ & & & I_{n-i-1}\end{array}\right)$

Moreover, the computation of determinant of Hessenberg matrix is equal for computing the determinant of pentadigonal centrosymmetric matrix. It means that the next step is how to compute Hessenberg matrix.

\subsection{Construct the Algorithm of Determinant Pentadiagonal Centrosymmetric Matrix}

Based on the study before about algorithm of determinant matrix, this determinant is constructed by using the two-term recuurence takes the following explanation.

First, take $n \times n$ matrix $\mathbf{Z}_{\mathbf{n}}=\left(\begin{array}{cc}\mathbf{P}_{\mathrm{n}-1} & \mathbf{q}_{\mathrm{n}-1} \\ \mathbf{r}^{\mathrm{T}}{ }_{\mathrm{n}-1} & \mathbf{S}_{\mathrm{n}}\end{array}\right)$, where $\mathbf{Z}_{\mathbf{n}-\mathbf{1}}$ has $(n-1) \times(n-1), \mathbf{q}_{\mathbf{n}-\mathbf{1}}, \mathbf{s}_{\mathbf{n}}$ are the scalar and $\mathbf{r}^{\mathrm{T}}{ }_{\mathrm{n}-1}$ has $1 \times(n-1)$ as size of this block matrix. Next, the determinant of $\mathbf{Z}_{\mathbf{n}}$ recursivelly is written as [14] :

$$
\begin{aligned}
& \mathbf{f}_{0}=1 \\
& \mathbf{f}_{\mathrm{i}}=\boldsymbol{\alpha}_{\mathrm{i}} \mathbf{f}_{\mathrm{i}-1} \text {, where } \boldsymbol{\alpha}_{1}=\mathbf{d}_{1}, \boldsymbol{\alpha}_{\mathrm{i}}=\mathbf{s}_{\mathrm{i}}-\mathbf{r}_{\mathrm{i}-1}{ }^{\mathrm{T}} \mathbf{Z}_{\mathrm{i}-1}{ }^{-1} \mathbf{q}_{\mathrm{i}-1}
\end{aligned}
$$
Generally, the determinant of $\mathbf{Z}_{\mathbf{i}}$ matrix is written by $\operatorname{det}\left(\mathbf{Z}_{\mathbf{i}}\right)=\mathbf{f}_{\mathbf{i}}$, where $i=1,2, \cdots, n$. 
Futheremore, by applying previous algorithm at sparse Hessenberg matrix having the following recursive.

$\boldsymbol{\alpha}_{n}=\mathbf{d}_{\mathrm{n}, \mathrm{n}}-\mathbf{h}_{\mathrm{n}, \mathrm{n}-1} \mathbf{e}_{\mathrm{n}-1}^{\mathrm{T}} \mathbf{H}^{-1}{ }_{\mathrm{n}-1}\left(\mathbf{d}_{\mathrm{n}-2, \mathrm{n}} \mathbf{e}_{\mathrm{n}-2}+\mathbf{d}_{\mathrm{n}-1, \mathrm{n}} \mathbf{e}_{\mathrm{n}-1}\right)$

$\boldsymbol{\alpha}_{n}=\mathbf{d}_{n, n}-d_{n, n-1}\left(d_{n-2, n} e^{T}{ }_{n-1} H^{-1}{ }_{n-1} e_{n-2}+d_{n-1, n} e^{T}{ }_{n-1} H^{-1}{ }_{n-1} e_{n-1}\right)$

, where $\mathbf{e}$ is vector unit.

By subtitute the equations

$$
\begin{aligned}
& \mathbf{e}^{\mathrm{T}}{ }_{\mathrm{n}-1} \mathbf{H}^{-1}{ }_{\mathrm{n}-1} \mathbf{e}_{\mathrm{n}-2}=-\frac{\mathbf{f}_{\mathrm{n}-3}}{\mathbf{f}_{\mathrm{n}-1}} \mathbf{d}_{\mathrm{n}-1, \mathrm{n}-2} \text { and } \\
& \mathbf{e}^{\mathrm{T}}{ }_{\mathrm{n}-1} \mathbf{H}^{-1}{ }_{\mathrm{n}-1} \mathbf{e}_{\mathrm{n}-1}=-\frac{\mathbf{f}_{\mathrm{n}-2}}{\mathbf{f}_{\mathrm{n}-1}} \text { then become } \\
& \boldsymbol{\alpha}_{n}=\mathbf{d}_{\mathrm{n}, \mathrm{n}}-\mathbf{d}_{\mathrm{n}, \mathrm{n}-1}\left(\frac{\mathbf{f}_{\mathrm{n}-3}}{\mathbf{f}_{\mathrm{n}-1}} \mathbf{d}_{\mathrm{n}-1, \mathrm{n}-2} \mathbf{d}_{\mathrm{n}-2, \mathrm{n}}-\frac{\mathbf{f}_{\mathrm{n}-2}}{\mathbf{f}_{\mathrm{n}-1}} \mathbf{d}_{\mathrm{n}-\mathbf{1 , n},}\right) .
\end{aligned}
$$

Moreover, by multiply the equation with $\mathbf{f}_{n-1}$ we have

$\mathbf{f}_{n}=\alpha_{n} \mathbf{f}_{n-1}=f_{n-1} d_{n, n}+d_{n, n-1}\left(f_{n-3} d_{n-1, n-2} d_{n-2, n}-f_{n-2} d_{n-1, n}\right)$

For a final step, construction of the algorithm of determinant pentadiagonal centrosymmetric matrix written as

$$
\begin{aligned}
& \mathbf{f}_{1}=\mathbf{d}_{11} \\
& \mathbf{f}_{2}=\mathbf{f}_{1} \mathbf{d}_{22}-\mathbf{d}_{21} d_{12} \\
& \mathbf{f}_{3}=\mathbf{f}_{2} \mathbf{d}_{33}+\mathbf{d}_{32}\left(\mathbf{d}_{21} \mathbf{d}_{13}-\mathbf{f}_{1} \mathbf{d}_{23}\right) \\
& \text { for } i=4,5, \cdots, n \\
& \mathbf{f}_{i}=f_{i-1} d_{, i}+d_{i, i-1}\left(f_{i-3} d_{i-1, i-2} d_{i-2, i}-f_{i-2} d_{i-1, i}\right) \\
& \text { end } \\
& \operatorname{det}(\mathbf{D})=\mathbf{f}_{\mathbf{n}} \text {. }
\end{aligned}
$$

This algorithm shows the rule of determinant of Hessenberg matrix can be contructed for general determinant of pentadiagonal centrosymmetric matrix.

To sum up of our work, the following of construction of the algorithm of determinant of pentadiagonal centrosymmetric matrix is proposed as :

Input : Pentadiagonal Matrix D (1)

Output : $\operatorname{det}(\mathbf{D})$

Step 1 Transform Sparse Hessenberg Matrix

- Propose Pentadiagonal Centrosymmetric Matrix (5)

- Form Sparse Hessenberg Matrix (8)

Step 2 Construct the Algorithm of Determinant

Pentadiagonal Centrosymmetric Matrix

- Applying Determinant of Sparse Hessenberg Matrix

Compute $\operatorname{det}(\mathbf{D})=\mathbf{f}_{\mathbf{n}}$

\section{CONCLUSION}

The algorithm of sparse Hessenberg matrix is applied on constructing the algorithm of determinant general pentadiagonal centrosymmetric matrix. This algorithm is used caused by same structure of main matrix is sparse Hessenberg matrix, therefore it will be applicable.

\section{ACKNOWLEDGMENTS}

The author grateefully acknowledge Miss Annisa Nur Latifah for her help in editing the paper and the technical assistance.

\section{REFERENCES}

[1] Xi-Le Zhao, On the inverse of a general pentadiagonal matrix, Applied Mathematics and Computation, 2008, pp. 639-646. DOI : https://doi.org/10.1016/j.amc.2008.03.004

[2] Tomohiro Sogabe, On a two-term recurrence for the determinant of a general matrix Appl. Math. Comput., 2007, pp. 785-788. DOI: https://doi.org/10.1016/j.amc.2006.08.156

[3] M. El-Mikkawy, A fast algorithm for evaluating nth order tridiagonal deter- minants J. Comput. Appl. Math., 2004, pp. pp. 581-584. DOI : https://doi.org/10.1016/j.cam.2003.08.044

[4] Tomohiro Sogabe, A fast numerical algorithm for the determinant of a pentadiagonal matrix Appl. Math. Comput., 2008, pp. 835-841. DOI : https://doi.org/10.1016/j.amc.2007.07.015

[5] Abderraman Marrero and V Tomeo, Some results on determinants and inverses of nonsingular pentadiagonal matrices J. Comput. Appl. Math., 2015, pp. 447-455. DOI : https://doi.org/10.1016/j.cam.2014.03.016

[6] Xiao-Guang Lv, Ting-Zhu Huang and Jiang Le, A note on computing the inverse and the determinant of a pentadiagonal Toeplitz matrix, Applied Mathematics and Computation, 2008, pp. 327-331 Contents.

DOI https://doi.org/10.1016/j.amc.2008.09.006

[7] Jiteng Jia, Boting Yang and Sumei Li, On a homogenous reccurence relation for the determinants of general pentadiagonal Toeplitz matrices Comp. Math Appl., 2016, pp. 1036-1044. DOI : https://doi.org/10.1016/j.camwa.2016.01.027

[8] Jiteng Jia and Sumei Li, On determinants of cyclic pentadiagonal matrices with Toeplitz structure 
Comp. Math Appl., 2017, pp. 304-309. DOI : https://doi.org/10.1016/j.camwa.2016.11.031

[9] Jolanta Borowska, Lena Lacinska and Jowita Rychlewska, On determinant of certain pentadiagonal matrix J. Appl. Math. Comput. Mech., 2013, pp. 21-26. DOI : 10.17512/jamcm.2013.3.03

[10] D.J. Evans, A recursive algorithm for determining the eigenvalues of a quindiagonal matrix Comput. J., 1973, pp. 70-73. DOI : https://doi.org/10.1093/comjnl/18.1.70

[11] R.A. Sweet, A recursive relation for the determinant of a pentadiagonal matrix Comm. ACM, 1969, pp. $330-332$.

DOI https://dl.acm.org/doi/10.1145/363011.363152

[12] Datta and Morgera, On the reducibility of centrosymmetric matrices-Aplication in engineering problems Circuits System Signal Process, 1989, pp. 71-95. DOI : https://doi.org/10.1007/BF01598746

[13] N Khasanah, Farikhin and B Surarso, The algorithm of determinant of centrosymmetric matrix based on lower Hessenberg form IOP Conf. Series : Journal of Physics : Conf. Series, 2017, 824. DOI : doi:10.1088/1742-6596/824/1/012028

[14] Di Zhao and Hongyi Li, On the computation of inverse and determinant of a kind of special matrices Appl. Math. Comput., 2015, pp. 721-726. DOI : https://doi.org/10.1016/j.amc.2014.09.114

[15] Y.-H. Chen, C.-Y. Yu, A new algorithm for computing the inverse and the determinant of a Hessenberg matrix Appl. Math. Comput., 2011, pp. 4433-4436. DOI https://doi.org/10.1016/j.amc.2011.10.022

[16] Nur Khasanah, Bayu Surarso, and Farikhin, Necessary and sufficient on the computation of determinant of a kind of special matrix AIP Conference Proceedings, 2022234 040014. DOI : https://doi.org/10.1063/5.0008498

[17] Zhong-Yun Liu, Some properties of centrosymmetric matrices Appl. Math. Comput., 2003, pp. 297-306. DOI : https://doi.org/10.1016/S0096-3003(02)00254-0

[18] Gene H. Golub and Charles F. Van Loan, 1996, Matrix Computations, third.ed., Johns Hopkins University Press : Baltimore and London 